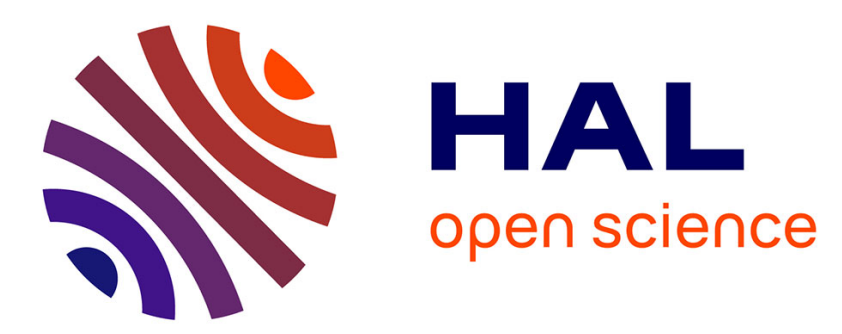

\title{
Convex regularizations for the simultaneous recording of room impulse responses
}

Alexis Benichoux, Laurent S. R. Simon, Emmanuel Vincent, Rémi Gribonval

\section{To cite this version:}

Alexis Benichoux, Laurent S. R. Simon, Emmanuel Vincent, Rémi Gribonval. Convex regularizations for the simultaneous recording of room impulse responses. IEEE Transactions on Signal Processing, 2014, 10.1109/TSP.2014.2303431 . hal-00934941

\section{HAL Id: hal-00934941 \\ https://hal.inria.fr/hal-00934941}

Submitted on 22 Jan 2014

HAL is a multi-disciplinary open access archive for the deposit and dissemination of scientific research documents, whether they are published or not. The documents may come from teaching and research institutions in France or abroad, or from public or private research centers.
L'archive ouverte pluridisciplinaire HAL, est destinée au dépôt et à la diffusion de documents scientifiques de niveau recherche, publiés ou non, émanant des établissements d'enseignement et de recherche français ou étrangers, des laboratoires publics ou privés. 


\title{
Convex regularizations for the simultaneous recording of room impulse responses
}

\author{
Alexis Benichoux, Laurent S. R. Simon, Emmanuel Vincent and Rémi Gribonval
}

\begin{abstract}
We propose to acquire large sets of room impulse responses (RIRs) by simultaneously playing known source signals on multiple loudspeakers. We then estimate the RIRs via a convex optimization algorithm using convex penalties promoting sparsity and/or exponential amplitude envelope. We validate this approach on real-world recordings. The proposed algorithm makes it possible to estimate the RIRs to a reasonable accuracy even when the number of recorded samples is smaller than the number of RIR samples to be estimated, thereby leading to a speedup of the recording process compared to state-of-the-art RIR acquisition techniques. Moreover, the penalty promoting both sparsity and exponential amplitude envelope provides the best results in terms of robustness to the choice of its parameters, thereby consolidating the evidence in favor of sparse regularization for RIR estimation. Finally, the impact of the choice of the emitted signals is analyzed and evaluated.
\end{abstract}

Index Terms-Room impulse response recording, convex optimization, compressed sensing

\section{INTRODUCTION}

The estimation of room impulse responses (RIRs) is a central problem in audio signal processing, in particular for spatial audio rendering and listening room equalization applications. The calibration of modern rendering systems such as wavefield synthesis (WFS) [1] requires the knowledge of the RIRs between the loudspeakers and several possible listener positions in order to compensate for the room effect [2]. For example, the study in [3] considered a WFS system of 48 loudspeakers and 6 multi-actuator panels calibrated in 96 different microphone positions. Similarly, typical recording of binaural room impulse responses (BRIRs) involves the acquisition of RIRs in up to several hundred source and listener spatial configurations [4]. For such applications, the RIRs must be estimated offline and they are subsequently kept fixed. Highly accurate estimation of the RIRs is unnecessary due in particular to their variation with temperature [5].

A larger number of loudspeakers or microphone positions would be welcome in many settings, but it is limited so far due in particular to the large total recording time implied by state-of-the-art RIR acquisition techniques, up to 1 hour or more [6], which is inconvenient in real-world scenarios. A speedup method using moving microphones [7] has been proposed for the collection of head related transfer functions (HRTF). The method proposed here focuses on RIRs instead and it is suitable for practical set ups such as, e.g., operas and theaters, where recording time is costly due to lack of room availability and high rental and salary costs, while additional computational time can be afforded for the estimation of the RIRs from the recordings.

While standard techniques typically consist of activating each loudspeaker in turn, we propose in this paper a way to record RIRs from multiple simultaneously active loudspeakers. We introduce a convex optimization algorithm for RIR estimation which exploits convex penalties on the RIRs in the spirit of compressed sensing [8]. We consider the classical $\ell_{1}$ sparsity-promoting penalty [9], [10], [11], [12], [13] as well as new penalties accounting for the fact that RIRs have an exponentially decaying envelope [14] . The techniques in [12] [13] and [10] for mixing filter estimation assume each source to be active alone in a certain time interval. Once this time interval has been localized, the corresponding filters are estimated using a subspace method [12], or convex optimization [10], [13]. Alternative Convolutive Independent Component Analysis techniques [15] assume the number of sources to be at most equal to the number of sensors. Our work is to our knowledge the first to get rid of these two assumptions. Our algorithm makes it possible to estimate the RIRs to a reasonable accuracy from an amount of recorded data that would otherwise be insufficient to estimate them at all, thereby leading to a speedup of the recording process.

In our preliminary study [16], we validated this approach on a set of synthetic RIRs using speech and Gaussian emitted signals and assuming exact knowledge of the room reverberation time. In this paper, we perform experiments on realworld recordings instead and we analyze both the choice of the emitted signals and the robustness of the algorithm to the values of its parameters.

The paper is organized as follows. In Section II] we formalize the considered problem and review existing techniques for individual and simultaneous measurement of RIRs. In Section III, we study the characteristics of RIRs in order to design appropriate penalties. We describe the linear system corresponding to the simultaneous recording of RIRs, and the convex optimization algorithm used for its inversion. Section IV describes the real-world acoustic setup used for the experiments and Section $\mathrm{V}$ analyzes the results. We conclude in Section VI

\section{EXISTING METHODS FOR INDIVIDUAL AND SIMULTANEOUS MEASUREMENT OF RIRS}

\section{A. Formalization of the problem}

The considered problem is formalized as follows. A set of $N$ loudspeakers simultaneously emit $N$ known discrete-time source signals $S_{n}(t)$ of duration $T$. Recording is performed using $M$ microphones, leading to $M$ discrete-time observed signals $X_{m}(t)$ of length $T$. The playback and recording processes are assumed to start at the same time. Assuming quasilinear behavior of both the loudspeakers and the microphones, 
the recorded signals are classically modeled as

$$
X_{m}(t)=\sum_{n=1}^{N}\left(A_{m n} \star_{[0, T-I]} S_{n}\right)(t)+E_{m}(t)
$$

where $A_{m n}(k)$ is the filter (RIR) of length $K$ between source $n$ and microphone $m, E_{m}(t)$ represents the background noise and the nonlinear contribution of the system, and $\star_{[0, T-I]}$ denotes convolution truncated to the discrete time interval $\llbracket 0, T-1 \rrbracket$ as defined in the Appendix.

In the following, we shall always assume that the emitted signals are centered and normalized according to their maximum amplitude, i.e., $\|\mathbf{S}\|_{\infty}=1$. As shorthands we will denote by $\mathbf{X} \in \mathbb{R}^{M \times T}$ the matrix of recorded signals, $\mathbf{S} \in \mathbb{R}^{N \times T}$ the matrix of emitted sources, $\mathbf{A} \in \mathbb{R}^{M \times N \times K}$ the array of RIRs and $\mathbf{E} \in \mathbb{R}^{M \times T}$ the matrix of noise samples. Using a matrix convolution notation, the recording process becomes

$$
\mathbf{X}=\mathbf{A} \star_{[, T-I]} \mathbf{S}+\mathbf{E} .
$$

The objective is to estimate the RIRs A. It can be decomposed into two complementary problems:

- estimate $\mathbf{A}$ given the set of emitted signals $\mathbf{S}$ and a set of recorded signals $\mathbf{X}$,

- design the set of emitted signals $\mathbf{S}$ so as to maximize the estimation accuracy.

The estimation problem is a linear inverse problem consisting in finding $\mathbf{A}$ that satisfies approximately the equality $\mathbf{X}=\mathbf{A} \star_{[0, T-I]} \mathbf{S}$. Assuming that the RIRs have a finite length $K$, the system is composed of $M T$ equations for $M N K$ unknown variables. Therefore it can be linearly inverted only if the recording duration in samples exceeds the critical recording duration

$$
T \geq T^{\text {crit }}:=N K .
$$

This is the overdetermined regime exploited by state-of-the-art RIR recording techniques as detailed below.

By contrast, the main contribution of this paper is to explore the regime where shorter recordings are targeted, i.e., $T<T^{\text {crit }}$. In this case the system is necessarily singular. Recovering the RIRs from the recordings becomes an underdetermined linear inverse problem, which requires non linear estimation techniques based on prior knowledge on the RIRs. The resulting measurement scheme can then be seen as a compressed sensing approach.

\section{B. State of the art}

1) Dirac impulses: The most straightforward way to measure RIRs is to emit Dirac pulses. Ideally, when measuring the RIR for a single source, the emitted signal has duration $D=1$ and is followed by silence for a recording duration $T=K$. For $N$ sources, $N$ Diracs are emitted every $K$ samples, so that the total recording duration is $T=N K=T^{\text {crit }}$.

In practice, electrical sparks, popping balloons, pistol and cannon shots have been used in the past to approximate Dirac pulses [17]. However with these techniques the shape and spectrum of the emitted signal is not well controlled, leading to imprecise RIR measurements. With modern digital equipment more controlled and reproducible Dirac pulses can be achieved, but these still yield RIR estimates with limited quality because of a poor signal-to-noise ratio (SNR).

The SNR of the recordings can be directly related to the root mean square (RMS) amplitude of the emitted signals

$$
\operatorname{RMS}(\mathbf{S})=10 \log _{10} \frac{\|\mathbf{S}\|_{2}^{2}}{T}
$$

expressed in decibels (dB). Dirac pulses have low RMS, due to the fact that most of the emitted signal consists of the silence following the impulses. Although recent studies have tried to adapt RIR estimation to particular types of impulses [18], the acoustic community often prefers signals with higher RMS as we shall see in the following.

A common technique to increase the SNR is to repeat the measurement $r$ times and to average the results. The resulting recording time is $T=r N K$.

2) Maximum length sequences $(M L S)$ : The MLS method introduced by Schroeder in 1979 [19] was initially designed to recover the RIR during an opera performance using an inaudible signal. A tutorial on both theoretical and practical aspects can be found in [20]. Besides having the greatest possible RMS, MLS signals exhibit two key properties: their autocorrelation function is close to a Dirac function, and their inverse in the sense of circular convolution is known in closed form.

MLS sequences $s \in \mathbb{R}^{D}$ are defined for lengths $D=2^{d}-$ 1 where $d \in \mathbb{N}$. The approximate decorrelation property of their circularly shifted versions allowed authors to conceive a simultaneous measurement technique provided that $D \geq N K$ [21]. The trick consists in sending simultaneously $N$ versions of the MLS: on the $n$-th loudspeaker, one sends the MLS sequence time-shifted by $n K$.

The emitted sequences may be periodically repeated every $D$ samples. Overall $r+2$ repeated periods make it possible to obtain $r$ noisy instances of the circularly convolved output that can be averaged to reduce the noise level. The first and last period can be truncated to emit only $K$ coefficients of the shifted sequences. The recording time achieved with this method is $T=r D+2 K \geq r N K+2 K$.

One problem is the constrained duration of the signal: in order to take advantage of the closed-form expression of the inverse, $D$ cannot be reduced to $N K$ unless $N K=2^{d}-1$ for some $d \in \mathbb{N}$. In addition, the nonlinearities of the speakers introduce artifact peaks in the measured RIR [22].

3) Exponential and linear sine sweeps: The latter limitation led to the introduction of sine sweep techniques by Farina in [23]. Their main advantage is that nonlinearities can easily be masked out from the recordings in the timefrequency domain. A sine sweep signal $s \in \mathbb{R}^{D}$ is defined by $s(t)=\sin \theta(t)$ where $\theta(t)$ is either exponential (exponential sweep) or quadratic (linear sweep). The typical sine sweep duration for RIR measurement is $1.5 \mathrm{~s}$ [24]. If the noise is stationary, doubling the sine sweep duration $D$ yields similar results as averaging $r=2$ sine sweeps. The inverse sweep has a closed form expression [25] but provides numerically less accurate RIR estimation than straightforward Fourier-domain inversion.

When measuring a single RIR with a sweep of duration $D$, the recording duration is typically $T=K+D$. A naive way to 
record $N$ RIRs is to successively emit $N$ sweeps of duration $D$, with a silence of duration $K$ between each, yielding a total duration $T=r N(D+K)$ in the case of $r$ repetitions.

A more clever way is to overlap the sine sweeps [26] such that their delayed versions are all disjoint in the timefrequency domain. Assuming quasi-linear behavior of both the loudspeakers and the microphones, a shift by $K$ samples is sufficient between two successive sweep ${ }^{1}$. When repeated $r$ times with overlapping sweeps of duration $D$, this leads to a recording duration $T=r N K+D$.

A disadvantage compared to MLS is that because high frequencies are present only at the end of the sweep, the emission must be padded with samples of silence in order to estimate the RIR at these frequencies. Together with the replacement of values in $\{-1,1\}$ by a sine function this results in a decrease of the RMS.

4) Role of the averaging: A measurement process typically involves an averaging step, in order to reduce the background noise. Usually the mean is taken among over $r=20$ instances [24], and sometimes up to $r=200$ [23] in the literature. A comparison between the durations of all methods is displayed in Table I. Simultaneous MLS techniques and overlapped sine sweeps result in a shorter recording duration than successive sine sweeps for large values of $r$.

In the rest of the paper, we present a faster technique and evaluate it for $r=1$ in order to bring the recording time down to a minimum. We decided not to perform averaging in order to present the shortest acceptable acquisition time. Nevertheless, it remains possible to apply this technique to the average of $r>1$ recordings.

To illustrate the potential savings in acquisition time, consider the setting of [21]. Using simultaneous MLS, $256 \times 12$ RIRs of length $500 \mathrm{~ms}$ have been recorded in $27.73 \mathrm{~min}$ instead of the naive $51.20 \mathrm{~min}$. The method proposed here would further drop the acquisition time down to $27.73 \times 0.45=$ $12.48 \mathrm{~min}$.

\begin{tabular}{|c|l|c|}
\hline & $T$ & RMS $(\mathrm{dB})$ \\
\hline Dirac & $r N K$ & $-10 \log _{10}(K)$ \\
Simultaneous MLS & $r N K+2 K$ & 0 \\
Successive Sweeps & $r N(K+D)$ & $\simeq-10 \log _{10}(2)$ \\
Overlapping sweeps & $r N K+D$ & $\simeq-10 \log _{10}(2)$ \\
\hline \hline Proposed & $<r N K$ & 0 \\
\hline
\end{tabular}

TABLE I

COMPARISON OF THE TOTAL RECORDING DURATION REQUIRED BY DIFFERENT RIR ACQUISITION TECHNIQUES.

\section{CONVEX OPTIMIZATION FRAMEWORK}

Earlier work on source separation [27] used convex optimization tools to estimate $\mathbf{S}$ given $\mathbf{X}$ when $\mathbf{A}$ is known, using a sparsity prior on the sources in the time-frequency domain. Here, we adapt the method in [27] to estimate $\mathbf{A}$ when $\mathbf{S}$ is known, by computing the minimizer of the following optimization problem

$$
\mathbf{A}_{0}=\min _{\mathbf{A}} \mathcal{P}(\mathbf{A}) \quad \text { s.t. } \quad \mathbf{X}=\mathbf{A} \star_{[0, T-I]} \mathbf{S}
$$

\footnotetext{
${ }^{1}$ The effect of nonlinearities on the choice of the shift is analyzed in [26].
}

where $\mathcal{P}(\mathbf{A})$ is a convex penalty function. To take into account the presence of background noise and small nonlinearities, it can be more relevant to solve a problem of the type

$$
\min _{\mathbf{A}} \mathcal{P}(\mathbf{A}) \quad \text { s.t. } \quad\left\|\mathbf{X}-\mathbf{A} \star_{[0, T-I]} \mathbf{S}\right\|_{2}^{2} \leq \varepsilon
$$

for some $\varepsilon>0$, which is known to be equivalent to the unconstrained minimization problem

$$
\mathbf{A}_{\lambda}=\operatorname{argmin}_{\mathbf{A}}\left\{\frac{1}{2}\left\|\mathbf{X}-\mathbf{A} \star_{[0, T-l]} \mathbf{S}\right\|_{2}^{2}+\lambda \mathcal{P}(\mathbf{A})\right\}
$$

for some Lagrangian parameter $\lambda>0$ [28, p. 664]. When the penalty $\mathcal{P}$ is convex, the limit when $\lambda$ tends toward zero provides the minimum of $\mathcal{P}$ subject to the equality constraint: $\lim _{\lambda \rightarrow 0} \mathbf{A}_{\lambda}=\mathbf{A}_{0}$.

\section{A. Choice of the penalties}

The choice of the penalty requires assumptions on the RIRs. Previous studies on dereverberation, source separation or RIR interpolation in a convex optimization framework have assumed that RIRs are formed by echoes at distinct instants, so that they are sparse [9], [10], [11]. This assumption is promoted by the non-weighted $\ell_{1}$ norm [29] which is often related to maximum a posteriori estimation with a Laplacian prior (see, e.g., [30]), although this relation is disputable [31].

The statistical theory of room acoustics [14] assumes instead that the samples of an RIR follow a Gaussian distribution, with an exponentially decaying amplitude envelope $\rho(t)$ depending on the size and the absorption coefficients of the surfaces of the room. Given the room reverberation time $\mathrm{RT}_{60}$ [14], that is the time required for a sound to decay $60 \mathrm{~dB}$ below its first reflection, the amplitude envelope is defined by

$$
\rho(t)=\sigma 10^{-3 t / \mathrm{RT}_{60}},
$$

where $\sigma$ is a scaling factor. We proposed in our preliminary study [16] to promote this behavior via weighted norms. Because $\mathrm{RT}_{60}$ is unknown a priori, it is set to an approximate value for the considered environment.

In order to assess the respective impact of the above two assumptions, we consider the following four penalties:

$$
\begin{aligned}
\mathcal{P}_{1}(\mathbf{A}) & =\left\|\frac{\mathbf{A}}{\sigma}\right\|_{1}=\sum_{m, n, k} \frac{\left|A_{m, n}(k)\right|}{\sigma} \\
\mathcal{P}_{2}(\mathbf{A}) & =\frac{1}{2}\left\|\frac{\mathbf{A}}{\sigma}\right\|_{2}^{2}=\sum_{m, n, k} \frac{\left|A_{m, n}(k)\right|^{2}}{2 \sigma^{2}} \\
\mathcal{P}_{1, \rho}(\mathbf{A}) & =\sum_{m, n, k} \frac{\left|A_{m, n}(k)\right|}{\rho(k)} \\
\mathcal{P}_{2, \rho}(\mathbf{A}) & =\sum_{m, n, k} \frac{\left|A_{m, n}(k)\right|^{2}}{2 \rho^{2}(k)}
\end{aligned}
$$

The penalties $\mathcal{P}_{1}$ and $\mathcal{P}_{1, \rho}$ promote sparsity while the penalties $\mathcal{P}_{2}$ and $\mathcal{P}_{2, \rho}$ do not, and the penalties $\mathcal{P}_{1, \rho}$ and $\mathcal{P}_{2, \rho}$ promote a decaying amplitude envelope while the penalties $\mathcal{P}_{1}$ and $\mathcal{P}_{2}$ do not. The solution of (5) with the penalty $\mathcal{P}_{2}$ is the naive Moore-Penrose pseudo-inverse [32, p. 257], which does not rely on any assumption on the RIRs. 
Note that we do not claim that real-world RIRs are actually sparse or that their amplitude envelope decays according to the assumed value of $\mathrm{RT}_{60}$, which is generally not true. We simply aim to evaluate the impact of these penalties on the RIR estimation accuracy. While pseudo-inversion is expected to perform well when the problem is overdetermined, we expect other penalties to yield better results in the underdetermined case even though the RIRs to be estimated do not satisfy these assumptions. This will be confirmed in Section $\mathrm{V}$

In our preliminary work [16], we had studied a fifth penalty $\mathcal{P}_{1,2, \rho}$ which is the sum of $\mathcal{P}_{1, \rho}$ up to a certain delay $K_{r}$ and $\mathcal{P}_{2, \rho}$ after that. This penalty was motivated by the physical observation that early echoes are sparse while reverberation is not. After evaluating it in the context of Section $\mathrm{V}$, we found that the best results are obtained when $K_{r}$ is either set to 0 , in which case $\mathcal{P}_{1,2, \rho}$ is equal to $\mathcal{P}_{2, \rho}$, or to $K$, in which case $\mathcal{P}_{1,2, \rho}$ is equal to $\mathcal{P}_{1, \rho}$. Due to this fundamental issue, we do not consider this penalty anymore hereafter.

\section{B. Convex optimization algorithm}

The optimization problem (7) has the form

$$
\min _{\mathbf{A}}\{\mathcal{L}(\mathbf{A})+\lambda \mathcal{P}(\mathbf{A})\},
$$

where $\mathcal{L}: \mathbf{A} \mapsto \frac{1}{2}\left\|\mathbf{X}-\mathbf{A} \star_{[O, T-l]} \mathbf{S}\right\|_{2}^{2}$ is a differentiable loss, $\nabla \mathcal{L}$ is Lipschitz and $\mathcal{P}$ is lower convex semi-continuous. To solve it, one can thus use the Fast Iterative Soft Thresholding Algorithm (FISTA) [33]. FISTA exploits the knowledge of the Lipschitz constant $L$ of the gradient $\nabla \mathcal{L}$ of the loss, as well as the proximal operator [34] of the penalty $\mathcal{P}$.

Definition 1: For $\mathcal{P}$ convex lower semi-continuous the proximal operator of $\mathcal{P}$ is the function

$$
\operatorname{prox}_{\mathcal{P}}: x \mapsto \operatorname{argmin}_{y}\left\{\mathcal{P}(y)+\frac{1}{2}\|x-y\|_{2}^{2}\right\}
$$

The general formulation of FISTA is given in Algorithm 1

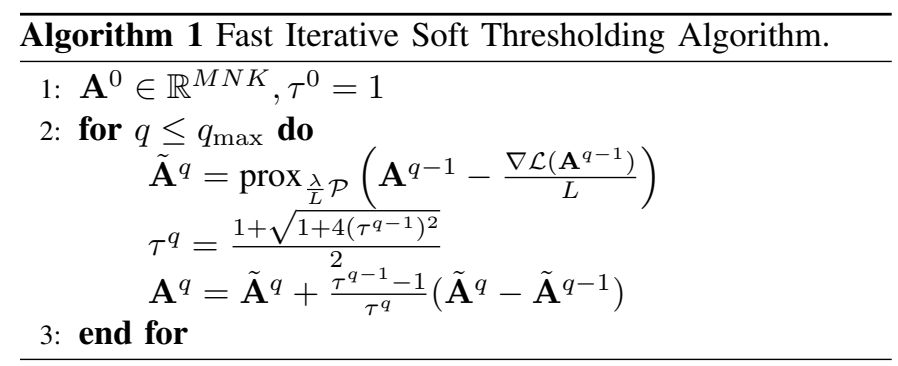

\section{Computing the proximal operators}

To fully specify the algorithm, we need to know the proximal operators of the penalties $\mathcal{P}_{i}$ introduced above. All penalties are separable, meaning that the operators can be processed coordinate by coordinate [35]. The penalties $\mathcal{P}_{1}$ and $\mathcal{P}_{1, \rho}$ are associated to weighted $\ell_{1}$ norms, and we obtain soft thresholding operators [27] as proximity operators. The proximity operators of $\mathcal{P}_{2}$ and $\mathcal{P}_{2, \rho}$, associated to squared weighted $\ell_{2}$ norms, can be obtained directly using differentiation.
Overall we obtain:

$$
\begin{aligned}
\operatorname{prox}_{\alpha \mathcal{P}_{1}}(\mathbf{A})_{m, n, k} & =\frac{A_{m n}(k)}{\left|A_{m n}(k)\right|}\left(\left|A_{m n}(k)\right|-\frac{\alpha}{\sigma}\right)^{+} \\
\operatorname{prox}_{\alpha \mathcal{P}_{2}}(\mathbf{A})_{m, n, k} & =\frac{A_{m n}(k)}{1+\frac{\alpha}{\sigma^{2}}} \\
\operatorname{prox}_{\alpha \mathcal{P}_{1, \rho}}(\mathbf{A})_{m, n, k} & =\frac{A_{m n}(k)}{\left|A_{m n}(k)\right|}\left(\left|A_{m n}(k)\right|-\frac{\alpha}{\rho(k)}\right)^{+}
\end{aligned}
$$

where ${ }^{+}$denotes the positive part of a real number.

\section{Gradient of the loss and its Lipschitz constant}

The computation of the gradient of $\mathcal{L}$ hinges on the introduction of the adjoint operator with respect to the truncated convolution. This construction is detailed in the Appendix.

Lemma 1: For $n \leq N$ we define $\mathbf{S}_{n}^{*} \in \mathbb{R}^{T}$ with $\mathbf{S}_{n}^{*}(t)=$ $S_{n}(T-t-1), 0 \leq t \leq T-1$, and $\mathbf{S}^{*}=\left(\mathbf{S}_{1}^{*}, \ldots, \mathbf{S}_{N}^{*}\right)$. We have

$$
\left\langle\mathbf{X}, \mathbf{A} \star_{[0, T-I]} \mathbf{S}\right\rangle=\left\langle\mathbf{X} \star_{[T-1, T+K-2]} \mathbf{S}^{*}, \mathbf{A}\right\rangle .
$$

The gradient can then be expressed as

$$
\nabla \mathcal{L}(\mathbf{A})=\left(\mathbf{X}-\mathbf{A} \star_{[0, T-I]} \mathbf{S}\right) \star_{[T T, T+K+-2]} \mathbf{S}^{*} .
$$

The Lipschitz constant $L$ of $\nabla \mathcal{L}$ is the modulus of the largest eigenvalue of the operator

$$
\mathbf{A} \mapsto\left(\mathbf{A} \star_{[0, T-1]} \mathbf{S}\right) \star_{[T-l, T+K-2]} \mathbf{S}^{*} .
$$

We compute it numerically using the power iteration algorithm [27, Algorithm 5].

\section{E. Pseudo-inversion for truncated RIRs}

Although the penalties $9 \sqrt{12}$ ) are mathematically motivated, it must be remembered that the RIR length is manually fixed to a certain length $K$ which is somewhat arbitrary. If we assume that only the first $K^{\prime}$ samples of the RIRs are nonzero with $K^{\prime} \leq \frac{T}{N}$, the system becomes overdetermined and the solution is more easily computed by pseudo-inversion instead:

$\mathbf{A}_{\text {cut }}=\min _{\mathbf{A}}\left\|\mathbf{X}-\mathbf{A} \star_{[0, T-I]} \mathbf{S}\right\|_{2}^{2} \quad$ s.t. $\quad \operatorname{supp}(\mathbf{A}) \subset \llbracket 0, K^{\prime}-1 \rrbracket$.

In order to make sure that the proposed penalties bring some benefit compared to simply shortening the assumed length of the RIRs, we also consider in the following the solution of 19) for $K^{\prime}=0.9 \frac{T}{N}$, where we found the overdeterminacy factor 0.9 to yield the best results experimentally. The first $K^{\prime}$ samples are computed using FISTA with the penalty $\mathcal{P}_{2}$ and with $\lambda \rightarrow 0$ and subsequently zero-padded to the total assumed length $K$. We will refer to this solution as $\mathcal{P}_{\text {cut }}$.

\section{EXPERIMENTAL STUDY}

In order to evaluate our approach, we conducted a set of experiments using real-world recordings. All the code and datasets involved have been made available for reproducibility 年

2 https://gforge.inria.fr/frs/?group_id=3390 


\section{A. Setup}

The recordings were made at IRISA, in the same room that was used to record certain signals of the Signal Separation Evaluation Campaign (SiSEC) [36]. The room is non rectangular and its dimensions are approximately $4 \times 5 \times 2.5 \mathrm{~m}$. The signals were emitted by $N=2,4,6,8$ coaxial loudspeakers. The recordings were captured with $M=10$ omnidirectional microphones. Both the sources and the microphones were randomly placed in the room. The sampling frequency was $44100 \mathrm{~Hz}$ both for playback and recording.

\section{B. Ground truth}

We first collected ground truth RIRs. The state-of-the-art choice is to use sine sweeps [24].

1) Acquisition process: We sent $r=20$ linear sine sweeps from $50 \mathrm{~Hz}$ to $22000 \mathrm{~Hz}$. Each sine sweep had a duration of $2 \mathrm{~s}$, and was followed by a silence of $1 \mathrm{~s}$. We then computed the average of these 20 recordings and estimated the RIRs by Fourier-domain inversion. Total duration of the acquisition process of the $N=8$ different sources is $480 \mathrm{~s}$.

2) Assumed duration $K$ of the RIRs: The obtained RIRs displayed a typical behavior: after a first part dominated by the direct path and first reflections, an exponentially decaying behavior was observed until the noise level was reached, after about $300 \mathrm{~ms}$. For this reason we chose to fix the length of the RIRs to $K=300 \mathrm{~ms}$ or 13230 samples.

3) Characterization of the background noise: The acquired recordings suffered from a strong low-frequency background noise, possibly due to air conditioning in the room. This prevented the evaluation of the estimated RIRs at these frequencies, since both the estimated and the ground truth RIRs were dominated by noise. For this reason, in the rest of the study, we chose to measure the estimation accuracy by comparing the high-frequency part of the estimated RIRs with that of the ground truth RIRs. Visualization of the spectrum of the noise suggested to keep all frequencies above $100 \mathrm{~Hz}$ for evaluation. Note that the full spectrum of the recorded signal is used for estimation nevertheless.

In this noisy set up, any method would fail to estimate the low frequency coefficients : it is most likely that the same experiment in better conditions would show an improvement even at low frequencies, following our first results on synthetic data [16].

\section{Performance measures}

We will use two categories of performance measures. A point wise comparison between the estimated filters and the ground truth provides a general fidelity metric, which is relevant for spatial audio reproduction. An additional equalization error metric allows us to evaluate the potential in terms of cross-channel cancellation.

1) Signal-to-noise ratio: The usual "noise level" metric employed for the assessment of RIR estimation is, as stated in [24], "the ratio expressed in $d B$ between the average power of the signal recorded by the microphone and the average power of the noise and distortions present in the tail of the deconvolved (linear) impulse response." This metric implicitly assumes that the difference between the estimated RIR and the true RIR is a stationary signal, so that the amount of noise and distortion in the tail is proportional to the total estimation error.

In the underdetermined context considered in this paper, the linear inverse problem (2) admits infinitely many solutions, most of which are completely inconsistent with this assumption. Therefore we chose a performance measure that reflects the estimation accuracy with respect to the ground truth. As a measurement of the error between the estimated RIRs $\hat{\mathbf{A}}$ and the ground truth RIRs A (in fact, the high-pass versions of $\hat{\mathbf{A}}$ and $\mathbf{A}$ as seen above), we propose to use the well-known SNR in $\mathrm{dB}$

$$
\operatorname{SNR}_{\mathbf{A}}(\hat{\mathbf{A}})=10 \log _{10} \frac{\|\mathbf{A}\|_{2}^{2}}{\|\hat{\mathbf{A}}-\mathbf{A}\|_{2}^{2}} .
$$

A frequency-wise $\mathrm{SNR}_{\mathbf{A}}$ is also performed Fig. 3, namely a $\mathrm{SNR}_{\mathbf{A}}$ between bands of the spectrum of $\hat{\mathbf{A}}$ and $\mathbf{A}$. It has been shown that thermal fluctuation induces errors in RIR measurements, such that the highest possible fidelity is around $25 \mathrm{~dB}$, depending on the sensor/source distance [37]. We will conduct in Section V-B2 a short qualitative study showing that a $\mathrm{SNR}_{\mathrm{A}}$ on the order of $15 \mathrm{~dB}$ is very satisfactory and that it corresponds for the chosen penalties to a "noise level" on the order of $50 \mathrm{~dB}$ as measured traditionally [24].

A first set of performance figures is given in Table [II where we compare the RIRs $\mathbf{A}_{r}$ estimated by averaging $r$ recorded sine sweeps to the ground truth $\mathbf{A}=\mathbf{A}_{20}$ obtained with $r=$ 20 .

\begin{tabular}{|l|ccccc|}
\hline Items averaged $r$ & 1 & 5 & 10 & 15 & 20 \\
\hline $\operatorname{SNR}_{\mathbf{A}}\left(\mathbf{A}_{r}\right)(\mathrm{dB})$ & 25.5 & 27.2 & 30.1 & 32.8 & $\infty$ \\
\hline
\end{tabular}

TABLE II

INFLUENCE OF AVERAGING ON THE SNR A OF THE SINE SWEEP METHOD.

While the $\mathrm{SNR}_{\mathrm{A}}$ quantifies the RIR estimation accuracy for a given estimation technique, it is also desirable to quantify the level of noise and nonlinear distortion present in the recorded signals from which the RIRs are estimated. For this, we use the SNR of the recording $\mathbf{X}$ (in fact, its high-pass version) defined as

$$
\operatorname{SNR}_{\mathbf{X}}(\mathbf{X}, \mathbf{S})=10 \log _{10} \frac{\left\|\mathbf{A} \star_{[0, T-l]} \mathbf{S}\right\|_{2}^{2}}{\left\|\mathbf{X}-\mathbf{A} \star_{[0, T-1]} \mathbf{S}\right\|_{2}^{2}}
$$

where $\mathbf{A}$ are the ground truth RIRs.

2) Equalization error: In the context of listening room compensation, the estimated filters must preserve interchannel properties in order to yield good cross-channel cancellation. Denoting by $\hat{\mathbf{A}}^{\dagger}(\omega)$ the pseudo inverse of the estimated mixing matrix at each frequency $\omega$, we measure the mean square equalization error using the following equalization error (EQE) metric:

$$
\operatorname{EQE}(\omega)=\frac{1}{M}\left\|I_{M}-\mathbf{A}(\omega) \hat{\mathbf{A}}^{\dagger}(\omega)\right\|_{2}^{2}
$$

where $\mathbf{A}(\omega)$ is the true mixing matrix, $I_{M}$ is the identity matrix of size $M$, and $\hat{\mathbf{A}}^{\dagger}(\omega)$ and $\mathbf{A}(\omega)$ are computed using the Fourier transform. The measured EQE is averaged over 
frequency. Assuming that the number of microphones $M$ is smaller than the number of loudspeakers $N$, the ideal case where $\hat{\mathbf{A}}$ perfectly matches $\mathbf{A}$ yields a zero EQE.

\section{Parameters of the proposed approach}

After collecting the ground truth RIRs, we made additional recordings within the same recording session and processed them via the proposed algorithm.

1) Source signals: Signals of different durations were emitted, including silence or not. Several recordings were made, for $N=2,4,6,8$ sources, and 3 types of signals :

- uniform random noise in $[-1,1]$;

- Bernoulli noise generated by a Bernouilli process on $\{-1,1\}$ with probability $p=\frac{1}{2}$;

- MLS sequences described above;

The emitted signals were normalized according to their maximum amplitude. In preliminary experiments we also tried human speech, the performance was much lower [38].

2) Parameters of the considered penalties: The scaling factor $\sigma$ for all penalties is set to $\sigma=1$. Given that nearoptimal performance is empirically obtained for $\lambda \rightarrow 0$, this parameter has in fact essentially no impact on the performance.

We consider different values of the reverberation parameter $R T_{60}$ in $\mathcal{P}_{1, \rho}, \mathcal{P}_{2, \rho}$ between $50 \mathrm{~ms}$ and 1 s. Fig. 1 shows two visualizations of one of the ground truth RIRs, rescaled to a maximum amplitude of 1 . The true value of the room reverberation time computed using Schroeder's backward integration method [39] is $R T_{60}=380 \mathrm{~ms}$. The assumption that the amplitude decays exponentially is clearly visible on the logarithmic view.
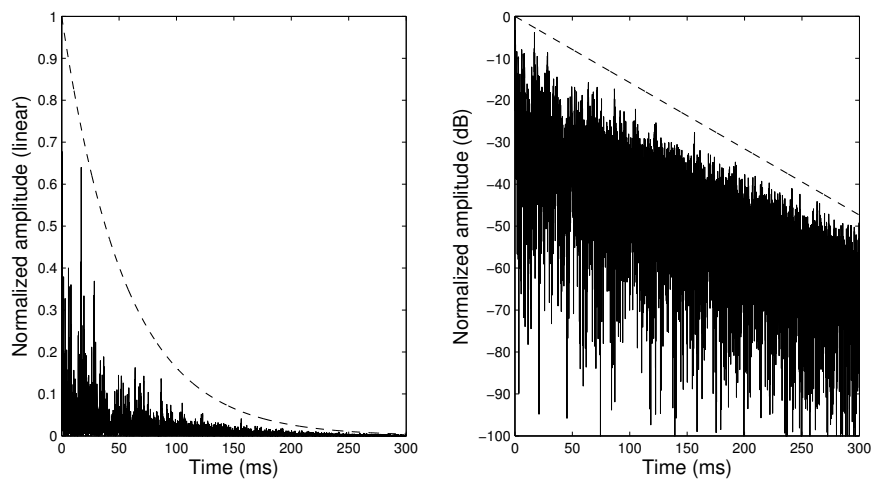

Fig. 1. Linear and logarithmic view of one ground truth RIR (plain) compared to the amplitude envelope $\rho$ (dashed) with $R T_{60}=380 \mathrm{~ms}$. The experiments in Section V-B1 will show that an exact value of $R T_{60}$ is not necessary to obtain good RIR estimates with the penalty $\mathcal{P}_{1, \rho}$.

3) Parameters of FISTA: The examination of Algorithm 1 and the expressions $(14,18)$ reveals that the variables relating to different microphones $m$ do not intervene with each other. This is consistent with the fact that the cost function (7) is additive with respect to $m$. Therefore, we equivalently apply FISTA to each microphone in turn.

The estimation of the Lipschitz constant requires 200 iterations of the power iteration algorithm. We know [40] that like many algorithms solving (7), FISTA requires a large number of iterations for small values of $\lambda$. In this situation we use the continuation trick also known as warm start : we run the algorithm for several decreasing values of $\lambda$ and initialize each run at the solution of the previous run. We run 16 instances of FISTA, using decreasing values $\lambda=\left\{10^{0}, \ldots, 10^{-15}\right\}$. The convergence of FISTA is observed for every $\lambda$ in about 500 iterations, we set $q_{\max }=2000$.

Theoretically, we expect to achieve the best results for a specific nonzero $\lambda$ but, given that the noisy low-frequency components are not taken into account in the performance measure, the noise level is low enough to neglect its influence and to consider the smallest value of $\lambda=10^{-15}$, which approximates the limit when $\lambda \rightarrow 0$. This will be confirmed experimentally in Section V-E

\section{EXPERIMENTAL RESULTS}

\section{A. Choice of the source signals}

1) Comparison between different types of sources: We first assess the impact of the choice of different source signals without silence in the case of an overdetermined system with $T=2 T^{\text {crit }}$ for $N=2$ sources, inverted using FISTA with $\mathcal{P}_{2}$ and with $\lambda \rightarrow 0$. Table [II shows the link between the RMS amplitude of the sources, the $\mathrm{SNR}_{\mathbf{X}}$ of the recording, and the $\mathrm{SNR}_{\mathbf{A}}$ of the estimated RIRs. Although Bernoulli and MLS signals potentially induce more nonlinearities than other signals, their higher RMS induces weaker noise, which altogether yields higher $\mathrm{SNR}_{\mathbf{X}}$ and $\left.\mathrm{SNR}_{\mathrm{A}}\right|^{3}$

\begin{tabular}{|l|ccc|}
\hline & Uniform & Bernoulli & MLS \\
\hline RMS (dB) & -4.8 & 0 & 0 \\
SNR $_{\mathbf{X}}(\mathrm{dB})$ & 17.1 & 18.2 & 18.3 \\
$\mathrm{SNR}_{\mathbf{A}}(\mathrm{dB})$ & 18.2 & 22.2 & 22.1 \\
\hline
\end{tabular}

TABLE III

RELATION BETWEEN RMS, SNR $\mathbf{X}$ AND SNR $\mathbf{A}$ FOR $T=2 T^{\text {CRIT }}$ DEPENDING ON THE CHOSEN SOURCE SIGNALS.

2) Influence of silence within the source signals: It is common in state-of-the-art methods to leave a silence between successive recordings, to make sure that the convolution is complete. However, including a silence of length $L$ within a signal of length $T$ decreases $\operatorname{SNR}_{\mathbf{X}}$ by up to $10 \log _{10}(1-L / T)$ $\mathrm{dB}$. This quantity grows as the system becomes more underdetermined. As an example, for the setup studied in the next section with $T \simeq 2 K$, a silence of length $L=K$ would result in a loss of up to $6 \mathrm{~dB}$ of SNR $\mathrm{X}$. We found in a preliminary experiment that this resulted in a similar or even bigger loss of $\mathrm{SNR}_{\mathrm{A}}$. We will therefore use Bernoulli signals without silence in all the following experiments.

\section{B. Performance of the proposed method for $T=0.45 T^{\text {crit }}$}

1) Influence of the penalty: As an example of the results obtained in an underdetermined setting, we compare in Table IV the performance of different penalties with $T=544 \mathrm{~ms}=$ $0.45 T^{\text {crit }}$ for $N=4,6$ or 8 sources. This corresponds to a reduction of the recording time by a factor of 2.2 with

\footnotetext{
${ }^{3}$ We remind that $\mathrm{SNR}_{\mathbf{X}}$ and $\mathrm{SNR}_{\mathbf{A}}$ account for the effect of both nonlinearities and noise.
} 


\begin{tabular}{|c|ccccc|}
\hline $\mathrm{SNR}_{\mathbf{A}}$ & $\mathcal{P}_{1, \rho}$ & $\mathcal{P}_{2, \rho}$ & $\mathcal{P}_{1}$ & $\mathcal{P}_{2}$ & $\mathcal{P}_{\text {cut }}$ \\
\hline $\mathrm{N}=4$ & 15.0 & 15.8 & 12.4 & 0.0 & 12.0 \\
$\mathrm{~N}=6$ & 14 & 14.2 & 10.7 & 0.0 & 11 \\
$\mathrm{~N}=8$ & 13.0 & 10.4 & 6.2 & 0.0 & 10.2 \\
\hline
\end{tabular}

TABLE IV

RIR ACCURACY DEPENDING ON THE CHOSEN PENALTY FOR $T=0.45 T^{\text {CRIT }}$ FOR DIFFERENT NUMBER OF SOURCES.

\begin{tabular}{|c|ccccc|}
\hline SNR $_{\text {A }}$ & $\mathcal{P}_{1, \rho}$ & $\mathcal{P}_{2, \rho}$ & $\mathcal{P}_{1}$ & $\mathcal{P}_{2}$ & $\mathcal{P}_{\text {cut }}$ \\
\hline source 1 & 17.8 & 18.4 & 15.2 & 0 & 15.4 \\
source 2 & 12.1 & 13.1 & 9.1 & 0 & 8.4 \\
source 3 & 15.7 & 16.4 & 13.3 & -0.1 & 12.3 \\
source 4 & 14.4 & 15.3 & 12.0 & 0 & 11.8 \\
\hline
\end{tabular}

TABLE V

DETAILED RIR ACCURACY FOR EACH OF THE $N=4$ SOURCES DEPENDING ON THE CHOSEN PENALTY FOR $T=0.45 T^{\text {CRIT }}$

respect to the critical time $T^{\text {crit }}$, which is itself smaller than the recording time required by state-of-the-art methods (see Table I).

The overall performance decreases when we add sources, but the performance ranking of penalties do not depend on the number of sources. Moreover, a closer look at the individual performance on each source in the case $N=4$ in Table IV shows that relative performance of the penalties does not depend on the source, although the absolute performance depends on the sources, and is poorer for sources further from the microphone. Unsurprisingly in this setting, naive pseudo inversion via $\mathcal{P}_{2}$ completely fails. The unweighted $\ell_{1}$ norm $\mathcal{P}_{1}$ and the RIR shortening approach $\mathcal{P}_{\text {cut }}$ are able to recover the RIRs to a certain extent, which is a good result given that no knowledge of $\mathrm{RT}_{60}$ is needed. However, the best results achieved by the weighted norms $\mathcal{P}_{1, \rho}$ and $\mathcal{P}_{2, \rho}$ which provide a $\mathrm{SNR}_{\mathrm{A}}$ on the order of $15 \mathrm{~dB}$ for $N=4$ sources. This shows the importance of promoting an exponential decaying envelope via the penalty.

2) Qualitative analysis of the resulting RIRs: Fig. 22 depicts one of the RIRs estimated using $\mathcal{P}_{1, \rho}$ compared to the ground truth. The global shape of the RIR is well recovered down to $-50 \mathrm{~dB}$. The $\mathrm{SNR}_{\mathrm{A}}$ of $15 \mathrm{~dB}$ in Table IV therefore appears to correspond to a noise level of $50 \mathrm{~dB}$ in Fig. 2. as measured traditionally [24]. As a comparison, efforts made to accelerate the acquisition of HRTF reach an estimation between 10 and $15 \mathrm{~dB}$ [7]. A view of the frequency-wise $\mathrm{SNR}_{\mathrm{A}}$ on 8 octave bands of the spectrum Fig. 3 confirms the accuracy of the estimation. No high pass filtering is performed before the evaluation Fig. 3, and the low performance on the lowest band $(0 \mathrm{~Hz}-125 \mathrm{~Hz})$ is visible.

The cross-channel cancellation and equalization performance is closely related to the filter estimation accuracy [5]. In fact it has been shown in the particular context of steadystate echo cancellation that the best possible Echo Return Loss Enhancement (ERLE) is equal to the $\mathrm{SNR}_{\mathbf{A}}$ for low values : due to physical limitations, an estimation of the filters with a $\mathrm{SNR}_{\mathbf{A}}$ above $20 \mathrm{~dB}$ does not allow better cross-channel cancellation.
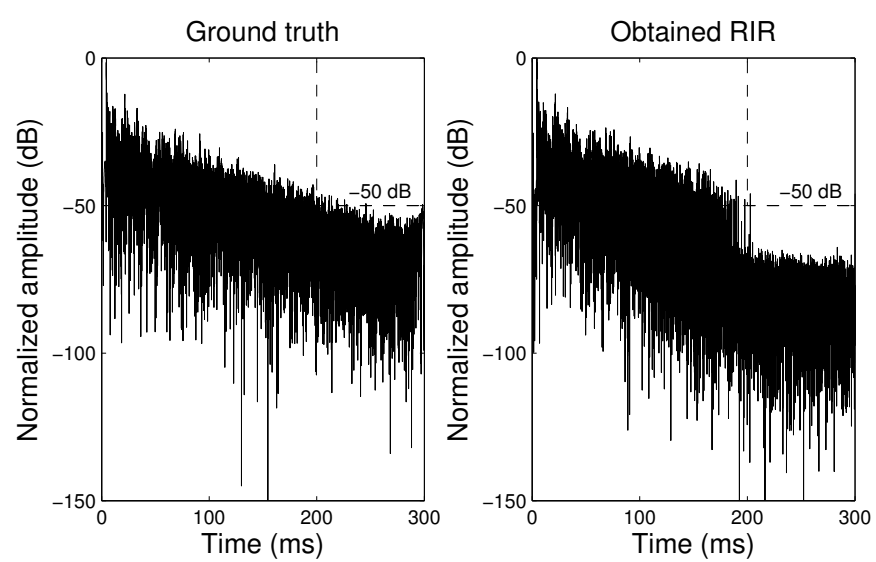

Fig. 2. Logarithmic view of one the RIRs estimated using $\mathcal{P}_{1, \rho}$ for $T=$ $0.45 T^{\text {crit }}$, compared to the ground truth.

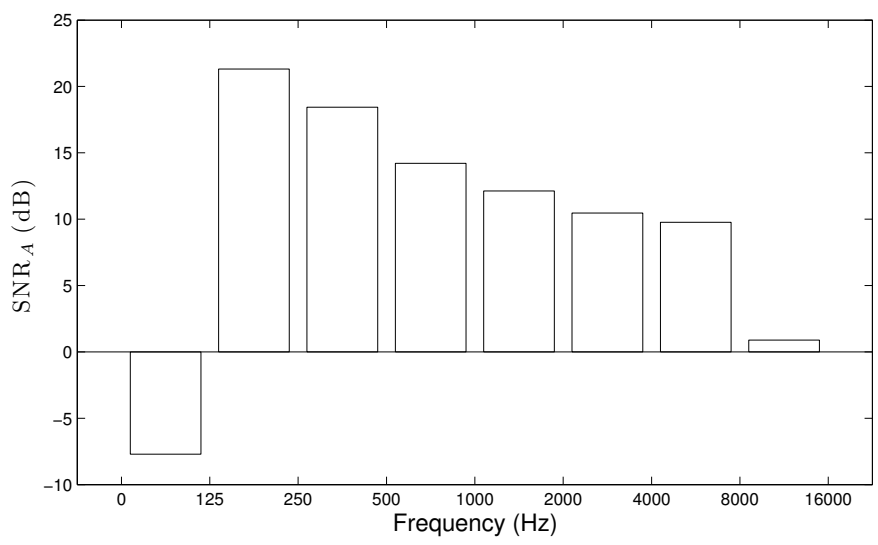

Fig. 3. Frequency wise $\mathrm{SNR}_{\mathrm{A}}$ between one the estimated RIRs of source 2 for $N=4, T=0.45 T^{\text {crit }}$ and the ground truth

\section{Robustness to an erroneous reverberation time}

The reverberation time is known only in retrospect, once the ground truth has been estimated. We wish to provide a reproducible method, therefore the best penalty has to provide a good performance estimation with the fewest required parameters. We show in this section that it is possible to use a rough estimate of the reverberation time. Fig. 4 bears witness to the robustness of the penalties to a bad guess of the room reverberation time $\mathrm{RT}_{60}$. The weighted $\ell_{2}$ penalty $\mathcal{P}_{2, \rho}$ performs best for any $\mathrm{RT}_{60}$ between $150 \mathrm{~ms}$ and $600 \mathrm{~ms}$. However, its performance drops quickly above that value. By contrast, the weighted $\ell_{1}$ penalty $\mathcal{P}_{1, \rho}$, which promotes both sparsity and exponential amplitude envelope, exhibits remarkable robustness and performs similarly or better than $\mathcal{P}_{2, \rho}$ for all $\mathrm{RT}_{60}$. For this reason, we select $\mathcal{P}_{1, \rho}$ as the best penalty in the remaining experiments.

\section{$D$. Influence of the recording time $T$}

Fig. 5 shows the performance as a function of the recording length $T$, where $T^{\text {crit }}=1200 \mathrm{~ms}$. While the performance of $\mathcal{P}_{2}$ is consistently low, that of $\mathcal{P}_{1, \rho}$ and $\mathcal{P}_{\text {cut }}$ appear to degrade gracefully as the recording time decreases. For instance, $\mathcal{P}_{1, \rho}$ still allows the recovery of the RIRs with more than $10 \mathrm{~dB}$ 


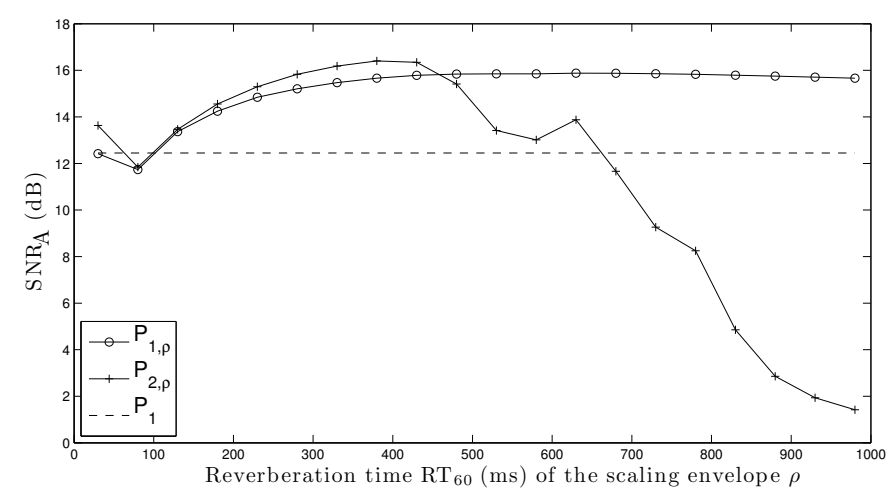

Fig. 4. Influence of the parameter of the amplitude envelope on the RIR accuracy for $T=0.45 T^{\text {crit }}$.

of $\mathrm{SNR}_{\mathbf{A}}$, with $T=300 \mathrm{~ms}=0.25 T^{\text {crit }}$, which corresponds to a reduction of the recording time by a factor of 4 . Note also that $\mathcal{P}_{1, \rho}$ outperforms $\mathcal{P}_{\text {cut }}$ as soon as $T \lesssim 0.6 T^{\text {crit }}$, and performs as well otherwise.

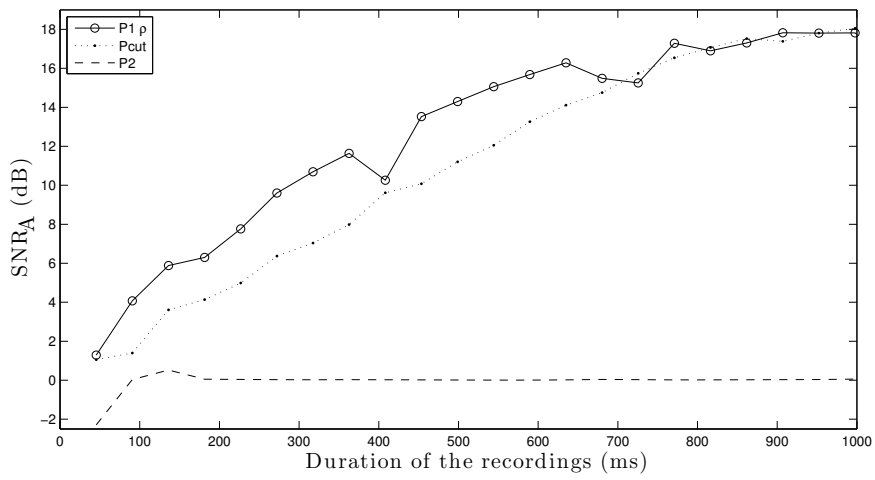

Fig. 5. $\mathrm{SNR}_{\mathbf{A}}$ of the different penalties as a function of the recording length $T$.

\section{E. Choice of the Lagrangian parameter $\lambda$}

While all the above results have been shown for $\lambda \rightarrow 0$, we expect that the best results are achieved for a specific nonzero $\lambda$ due to the presence of noise and nonlinearities. The analysis of the performance of $\mathcal{P}_{1, \rho}$ as a function of $\lambda$ in Fig. 6 shows that, as the system becomes more underdetermined, the gain obtained by choosing the optimal $\lambda$ becomes smaller. For $T=$ $0.45 T^{\text {crit }}$, a gain of about $0.5 \mathrm{~dB}$ is obtained for the optimal $\lambda=10^{-2}$. However, the decrease of performance is observed for larger values of $\lambda$. Although there is theoretically a link between $\lambda$ and the background noise level, there is no way to predict the optimal value of $\lambda$ to our knowledge. The choice $\lambda \rightarrow 0$ appears to be the most robust, and requires no oracle information.

\section{F. Equalization error}

Similarly to Table II] we computed the equalization error between $\hat{\mathbf{A}}=\mathbf{A}_{r}$ estimated by averaging $r$ recorded sine sweeps and the ground truth $\mathbf{A}=\mathbf{A}_{20}$ obtained with $r=20$, $M=1, N=4$. The results are displayed Table VI The results

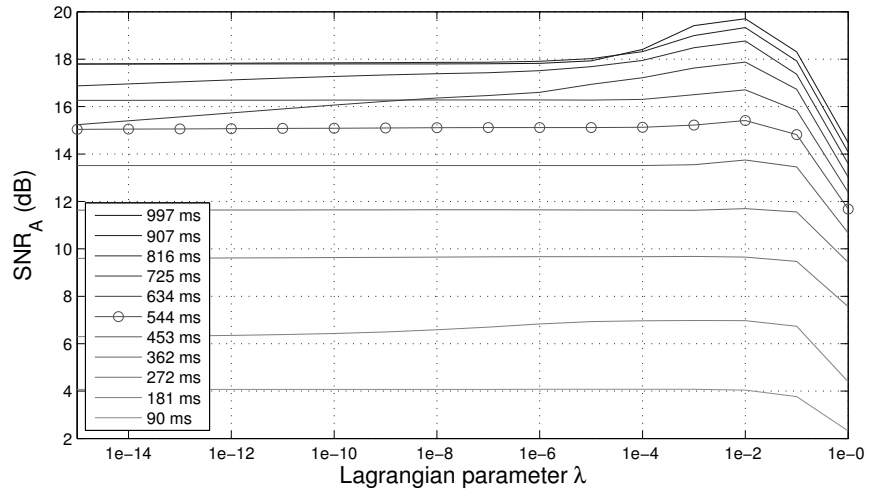

Fig. 6. Influence of the Lagrangian parameter $\lambda$ on the accuracy of the RIRs obtained with $\mathcal{P}_{1, \rho}$ for several recording lengths $T$.

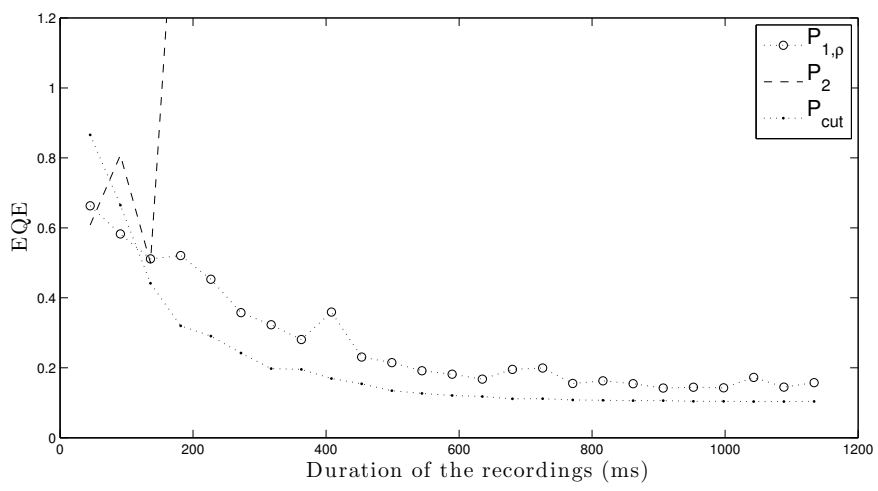

Fig. 7. EQE of the different penalties as a function of the recording length $T$.

for $r=1$ correspond to a recording duration of $9200 \mathrm{~ms}$. Fig. 7 shows the average equalization error EQE for different recording durations $T$, with $M=1, N=4$. First, we observe the failure of the naive pseudo inverse associated to $\mathcal{P}_{2}$ whose EQE remains well above 0.6 , in contrast to $\mathcal{P}_{1, \rho}$ and $\mathcal{P}_{\text {cut }}$. Second, we notice that for $T=T^{\text {crit }}=1200 \mathrm{~ms}, \mathcal{P}_{\text {cut }}$ achieves almost the same $\mathrm{EQE}$ as $\mathbf{A}_{r}$ with $r=1$, which required a recording time almost 8 times larger. Last, $\mathcal{P}_{\text {cut }}$ with $T=$ $600 \mathrm{~ms}=0.5 T^{\text {crit }}$ still yields comparable performance.

\section{G. Computational complexity}

Each iteration of the FISTA algorithm requires the computation of the gradient and a proximal operator. In our case the computationally heaviest operation is the convolution between the source and the filters, which requires

$$
O(M N \max (T, K) \log (\max (T, K)))
$$

operations. A theoretical estimation of the number of iterations needed to reach a target precision can be found in [33]. In

\begin{tabular}{|c|ccccc|}
\hline Items averaged $r$ & 1 & 5 & 10 & 15 & 20 \\
\hline Equalization error EQE & 0.0798 & 0.0580 & 0.0367 & 0.0190 & 0 \\
\hline
\end{tabular}

TABLE VI

INFLUENCE OF AVERAGING ON THE EQE OF THE SINE SWEEP METHOD. 
practice, we choose to set the number of iterations to 2000 . Using Matlab on a dual-core $3.40 \mathrm{GHz} \mathrm{CPU}$, the computation time is on the order of 20 min per microphone, per source and per second of recorded signal.

\section{CONCLUSION}

We proposed an algorithm to estimate RIRs from recordings of multiple active loudspeakers where the number of recorded samples is smaller than the number of RIR samples to be estimated. This algorithm relies on convex penalties incorporating knowledge about the RIRs. We investigated both existing and new penalties and showed that the penalty $\mathcal{P}_{1, \rho}$ promoting sparsity and exponential amplitude envelope is the most robust to an erroneous choice of the $\mathrm{RT}_{60}$. These two assumptions on the RIRs have hence been proven to be beneficial for the purpose of regularization, although actual RIRs do not satisfy them exactly.

Following the described framework, further experiments could be performed to expand this technique to other acoustic responses such as BRIRs. The estimation of RIRs is also an important problem in blind source separation, where they are called mixing filters. The proposed algorithm is a first brick towards a new algorithm for joint estimation of the source signals and the mixing filters which would make use of the proposed RIR regularization.

In addition, inter channel information is not used in this work: in fact, the RIRs associated to different microphones are estimated separately. In the spirit of the simultaneous sparse recovery of the filters designed for blind calibration [11], one could envision joint RIR models allowing a joint estimation with potentially shorter measurements. A complementary approach would be to exploit cross-channel relations to improve the filter estimation quality. Techniques based on such relations have recently been used on multi-channel dereverberation using sparsity constraints [41], but they are primarily designed for a mono-source setup. The main challenge would be to adapt them to the intrinsically multi-source context of our approach.

\section{APPENDIX}

The computation of $\nabla \mathcal{L}$ boils down to that of the adjoint operator of the truncated matrix convolution product $\star_{[0, T-I]}$.

The convolution with the RIR is causal. A convenient way to model its convolution is to see the signals in $\ell_{2}(\mathbb{Z})$, with a finite support. For $x, y \in \ell_{2}(\mathbb{Z})$ we denote by $*$ the standard convolution

$$
x * y(\tau)=\sum_{t \in \mathbb{Z}} x(t) y(\tau-t) .
$$

For $T \in \mathbb{N}$, we define the truncation operator:

$$
\begin{aligned}
\mathbb{R}^{\mathbb{Z}} & \longrightarrow \\
P_{T}^{*}:\left(x_{t}\right)_{t \in \mathbb{Z}} & \longmapsto\left(x_{t}\right)_{0 \leq t \leq T-1}
\end{aligned}
$$

and its adjoint, the double-sided zero-padding operator

$$
\begin{array}{ccc}
\mathbb{R}^{T} & \longrightarrow & \mathbb{R}^{\mathbb{Z}} \\
P_{T}:\left(x_{0}, \ldots, x_{T-1}\right) & \longmapsto & \left(\ldots 0, x_{0}, \ldots, x_{T-1}, 0, \ldots\right) .
\end{array}
$$

Now consider $\mathbf{x} \in \mathbb{R}^{T}, \mathbf{s} \in \mathbb{R}^{T}, \mathbf{a} \in \mathbb{R}^{K}$. The definition of the truncated convolution product $\star_{[0, T-I]}$ is

$$
\mathbf{a} \star_{[0, T-1]} \mathbf{s}=P_{T}^{*}\left(P_{K}(\mathbf{a}) * P_{T}(\mathbf{s})\right) .
$$

For $x, s, a \in \ell_{2}(\mathbb{Z})$, denoting $\bar{s}(t)=s(-t), t \in \mathbb{Z}$, we have:

$$
\langle x, a * s\rangle=\langle x * \bar{s}, a\rangle
$$

Then we can write

$$
\begin{aligned}
\left\langle\mathbf{x}, \mathbf{a} \star_{[0, T-I]} \mathbf{s}\right\rangle & =\left\langle\mathbf{x}, P_{T}^{*}\left(P_{K}(\mathbf{a}) * P_{T}(\mathbf{s})\right)\right\rangle \\
& =\left\langle P_{T}(\mathbf{x}), P_{K}(\mathbf{a}) * P_{T}(\mathbf{s})\right\rangle \\
& =\left\langle P_{T}(\mathbf{x}) * \overline{P_{T}(\mathbf{s})}, P_{K}(\mathbf{a})\right\rangle \\
& =\left\langle P_{K}^{*}\left(P_{T}(\mathbf{x}) * \overline{P_{T}(\mathbf{s})}\right), \mathbf{a}\right\rangle,
\end{aligned}
$$

where we used the notation of (27).

There remains to express $P_{K}^{*}\left(P_{T}(\mathbf{x}) * \overline{P_{T}(\mathbf{s})}\right)$ as a truncated convolution. Since $P_{T}(\mathbf{s})$ is supported on $\llbracket 0, T-1 \rrbracket$, its time reversed version $\overline{P_{T}(\mathbf{s})}$ is supported on $\llbracket-(T-1), 0 \rrbracket$. Define $\mathbf{s}^{*} \in \mathbb{R}^{T}$ by $\mathbf{s}^{*}(t):=\mathbf{s}(T-1-t), 0 \leq t \leq T-1$. We have $\overline{P_{T}(\mathbf{s})}=\delta_{-(T-1)} * P_{T}\left(\mathbf{s}^{*}\right)$, hence we can write

$$
\begin{aligned}
P_{K}^{*}\left(P_{T}(\mathbf{x}) * \overline{P_{T}(\mathbf{s})}\right) & =P_{K}^{*}\left(\delta_{-(T-1)} * P_{T}(\mathbf{x}) * P_{T}\left(\mathbf{s}^{*}\right)\right) \\
& =\mathbf{x} \star_{[T-1, T+K-2]} \mathbf{s}^{*}
\end{aligned}
$$

where the last equality comes from the fact that $P_{K}^{*}\left(\delta_{-(T-1)} *\right.$ $u)$ is the restriction of the sequence $u \in \ell_{2}(\mathbb{Z})$ to the interval $\llbracket T-1,(T-1)+(K-1) \rrbracket$.

The multichannel and multisource case $M, N \geq 1$ is now straightforward. For $1 \leq n \leq N$ we define $\mathbf{S}_{n}^{*} \in \mathbb{R}^{T}$ the time reversal of the source signal $\mathbf{S}_{n}$, i.e., for $0 \leq t \leq T-1$, $\mathbf{S}_{n}^{*}(t)=\mathbf{S}_{n}(T-1-t)$, and $\mathbf{S}^{*}=\left(\mathbf{S}_{1}^{*}, \ldots, \mathbf{S}_{N}^{*}\right)$. Using these notations and the previous computation the following holds

$$
\begin{aligned}
\left\langle\mathbf{X}, \mathbf{A} \star_{[0, T-T]} \mathbf{S}\right\rangle & =\left\langle\left(\begin{array}{c}
\mathbf{X}_{1} \\
\vdots \\
\mathbf{X}_{M}
\end{array}\right) \star_{[T-l, T+K-2]}\left(\mathbf{S}_{1}^{*}, \ldots \mathbf{S}_{N}^{*}\right), \mathbf{A}\right\rangle \\
& =\left\langle\mathbf{X} \star_{[T-l, T+K-2]} \mathbf{S}^{*}, \mathbf{A}\right\rangle .
\end{aligned}
$$

\section{REFERENCES}

[1] A. Berkhout, D. de Vries, and P. Vogel, "Acoustic control by wave field synthesis," Journal of the Acoustical Society of America, vol. 93, pp. 2764-2778, 1993.

[2] S. Spors, D. Seuberth, and R. Rabenstein, "Multiactuator panels for wave field synthesis: Evolution and present developments," Journal of the Audio Engineering Society, vol. 58, no. 12, pp. 1045-1063, 2010.

[3] E. Corteel, "Equalization in an extended area using multichannel inversion and wave field synthesis," Journal of the Audio Engineering Society, vol. 54, no. 12, pp. 1140-1161, 2006.

[4] H. Kayser, S. D. Ewert, J. Anemüller, T. Rohdenburg, V. Hohmann, and B. Kollmeier, "Database of multichannel in-ear and behind-the-ear head-related and binaural room impulse responses," EURASIP Journal on Advances in Signal Processing, vol. 2009, 2009, article ID 298605.

[5] M. E. Knappe and R. Goubran, "Steady-state performance limitations of full-band acoustic echo cancellers," in Proc. 1994 IEEE Int. Conf. on Acoustics, Speech, and Signal Processing (ICASSP), vol. 2, 1994, pp. 73-76.

[6] M. Ferrer, A. González, P. Zuccarello, and A. Camacho, "On the practical implementation of multichannel adaptive filters based on LMS, RLS, FTF and FAP algorithms for active control," in Proc. 10th Int. Congress on Sound and Vibration, 2004.

[7] T. Ajdler, L. Sbaiz, and M. Vetterli, "Dynamic measurement of room impulse responses using a moving microphone," Journal of the Acoustical Society of America, vol. 122, no. 3, pp. 1636-1645, 2007. 
[8] D. Donoho, "Compressed sensing," IEEE Transactions on Information Theory, vol. 52, no. 4, pp. 1289-1306, 2006.

[9] Y. Lin, J. Chen, Y. Kim, and D. Lee, "Blind channel identification for speech dereverberation using $\ell 1$-norm sparse learning," in Advances in Neural Information Processing Systems 20. MIT Press, 2007, pp. 921928.

[10] P. Sudhakar, S. Arberet, and R. Gribonval, "Double sparsity: Towards blind estimation of multiple channels," in Proc. 9th Int. Conf. on Latent Variable Analysis and Signal Separation (LVA/ICA). Springer, 2010, pp. 571-578.

[11] R. Mignot, L. Daudet, and F. Ollivier, "Compressed sensing for acoustic response reconstruction: interpolation of the early part," in Proc. 2011 IEEE Int. Workshop on Applications of Signal Processing to Audio and Acoustics (WASPAA), 2011, pp. 225-228.

[12] A. Aissa-El-Bey, K. Abed-Meraim, and Y. Grenier, "Blind separation of underdetermined convolutive mixtures using their time-frequency representation," IEEE Transactions on Audio Speech and Language Processing, vol. 15, no. 5, pp. 1540-1550, 2007.

[13] M. Yu, W. Ma, J. Xin, and S. Osher, "Multi-channel $\ell_{1}$ regularized convex speech enhancement model and fast computation by the split bregman method," IEEE Transactions on Audio, Speech and Language Processing, vol. 20, no. 2, pp. 661-675, 2012.

[14] H. Kuttruff, Room Acoustics, 4th ed. New York: CRC Press, 2000, no. 0419245804, pp 97-100.

[15] S. Makino, T. Lee, and H. Sawada, Blind speech separation. Springer, 2007.

[16] A. Benichoux, E. Vincent, and R. Gribonval, "A compressed sensing approach to the simultaneous recording of multiple room impulse responses," in Proc. 2011 IEEE Int. Workshop on Applications of Signal Processing to Audio and Acoustics (WASPAA), 2011, pp. 285-288.

[17] K. Bodlund, "On the use of the integrated impulse response method for laboratory reverberation measurements," Journal of Sound and Vibration, vol. 56, no. 3, pp. 341-362, 1978.

[18] J. S. Abel, N. Bryan, P. Huang, M. Kolar, and B. Pentcheva, "On estimating room impulse responses from recorded balloon pops," in Proc. 129th AES Convention, 2010, article ID 8171.

[19] M. Schroeder, "Integrated-impulse method measuring sound decay without using impulses," Journal of the Acoustical Society of America, vol. 66, pp. 497-500, 1979 .

[20] W. Chu, "Impulse-response and reverberation-decay measurements made by using a periodic pseudorandom sequence," Applied Acoustics, vol. 29, no. 3, pp. 193-205, 1990.

[21] A. Gonzalez, P. Zuccarello, G. Pinero, and M. de Diego, "Simultaneous measurement of multichannel acoustic systems," Journal of the Audio Engineering Society, vol. 52, no. 1/2, pp. 26-42, 2004.

[22] M. Wright, "Comments on aspects of MLS measuring systems," Journal of the Audio Engineering Society, vol. 43, no. 1, pp. 48-49, 1995.

[23] A. Farina, "Simultaneous measurement of impulse response and distortion with a swept-sine technique," in Proc. AES 108th Convention, 2000, pp. $18-22$

[24] G. Stan, J. Embrechts, and D. Archambeau, "Comparison of different impulse response measurement techniques," Journal of the Audio Engineering Society, vol. 50, no. 4, pp. 249-262, 2002.

[25] A. Novak, L. Simon, F. Kadlec, and P. Lotton, "Nonlinear system identification using exponential swept-sine signal," IEEE Transactions on Instrumentation and Measurement, vol. 59, no. 8, pp. 2220-2229, 2010.

[26] P. Majdak, P. Balazs, and B. Laback, "Multiple exponential sweep method for fast measurement of head-related transfer functions," Journal of the Audio Engineering Society, vol. 55, no. 7/8, pp. 623-637, 2007.

[27] M. Kowalski, E. Vincent, and R. Gribonval, "Beyond the narrowband approximation: Wideband convex methods for under-determined reverberant audio source separation," IEEE Transactions on Audio, Speech, and Language Processing, vol. 18, no. 7, pp. 1818-1829, 2010.

[28] S. Mallat, A Wavelet Tour of Signal Processing. Academic Press, 1999.

[29] D. Donoho, "For most large underdetermined systems of linear equations the minimal $\ell^{1}$-norm solution is also the sparsest solution," Communications on pure and applied mathematics, vol. 59, no. 6, pp. 797-829, 2006.

[30] S. Winter, W. Kellermann, H. Sawada, and S. Makino, "MAP-based underdetermined blind source separation of convolutive mixtures by hierarchical clustering and 11-norm minimization," EURASIP Journal on Applied Signal Processing, vol. 2007, no. 1, pp. 81-81, 2007.

[31] R. Gribonval, "Should penalized least squares regression be interpreted as maximum a posteriori estimation?" IEEE Transactions on Signal Processing, vol. 59, no. 5, pp. 2405-2410, 2010.
[32] G. Golub and C. F. Van Loan, Matrix computations (3rd ed.). Johns Hopkins University Press, 1996.

[33] A. Beck and M. Teboulle, "A fast iterative shrinkage-thresholding algorithm for linear inverse problems," SIAM Journal on Imaging Sciences, vol. 2, no. 1, pp. 183-202, 2009.

[34] J. Moreau, "Fonctions convexes duales et points proximaux dans un espace hilbertien," CR Acad. Sci. Paris Sér. A Math, vol. 255, pp. 28972899, 1962.

[35] P. Combettes and V. Wajs, "Signal recovery by proximal forwardbackward splitting," Multiscale Modeling and Simulation, vol. 4, no. 4, pp. 1168-1200, 2006.

[36] E. Vincent, S. Araki, F. J. Theis, G. Nolte, P. Bofill, H. Sawada, A. Ozerov, B. V. Gowreesunker, D. Lutter, and N. Q. K. Duong, "The Signal Separation Evaluation Campaign (2007-2010): Achievements and remaining challenges," Signal Processing, vol. 92, pp. 1928-1936, 2012.

[37] G. W. Elko, E. Diethorn, and T. Gänsler, "Room impulse response variation due to thermal fluctuation and its impact on acoustic echo cancellation," in Proc. 8th Int. Workshop on Acoustic Echo and Noise Control (IWAENC), 2003, pp. 67-70.

[38] A. Benichoux, E. Vincent, and R. Gribonval, "Optimisation convexe pour l'estimation simultanée de réponses acoustiques," in Actes du $23 e$ Colloque du Groupement de Recherche en Traitement du Signal et des Images (GRETSI), Bordeaux, France, May 2011, article ID 132.

[39] M. R. Schroeder, "New method of measuring reverberation time," Journal of the Acoustical Society of America, vol. 37, no. 3, pp. 409$412,1965$.

[40] I. Loris, "On the performance of algorithms for the minimization of $\ell_{1}$-penalized functionals," Inverse Problems, vol. 25, no. 3, pp. 35008 $35023,2009$.

[41] M. Yu and F. K. Soong, "Constrained multichannel speech dereverberation," in Proc. Interspeech, 2012.

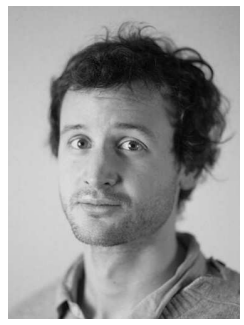

Alexis Benichoux is a Research Assistant at University of Southampton. He obtained his Ph.D. in Signal processing and Telecomunication at Université de Rennes I in 2013. His current research interests include algorithms for inverse problems with applications to audio source separation, room impulse response estimation, and brain network imaging.

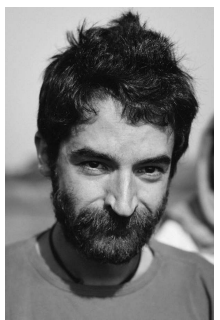

Laurent S. R. Simon is a Research Assistant at LIMSI (Orsay, France). He obtained his Ph.D. in Psychoacoustics from Institute of Sound Recording, at the University of Surrey (Guildford, United Kingdom) in 2011. From 2011 to 2013, he worked as a Postdoc at INRIA Rennes (Rennes, France). His research topics include Blind Audio Source Separation, Spatial Audio and Perceptual Audio Evaluation. $\mathrm{He}$ is now working on binaural listening experience evaluation. 


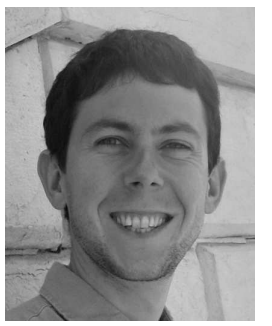

Emmanuel Vincent (M'07 - SM'10) is a Research Scientist with Inria (Nancy, France). He received the $\mathrm{Ph} . \mathrm{D}$. degree in music signal processing from IRCAM (Paris, France) in 2004 and worked as a Research Assistant with the Centre for Digital Music at Queen Mary, University of London (London, U.K.) from 2004 to 2006. His research focuses on probabilistic machine learning for speech and audio signal processing, with application to real-world audio source localization and separation, noise-robust speech recognition and music information retrieval.

$\mathrm{He}$ is a founder of the series of Signal Separation Evaluation Campaigns (SiSEC) and CHiME Speech Separation and Recognition Challenges. Dr. Vincent is an Associate Editor for the IEEE Transactions on Audio, Speech and Language Processing.

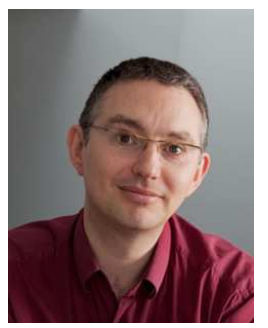

Rémi Gribonval studied at École Normale Supérieure, Paris, France, from 1993 to $1997 . \mathrm{He}$ received the $\mathrm{Ph}$. D. degree in applied mathematics from the University of Paris-IX Dauphine, Paris, France, in 1999, and his Habilitation à Diriger des Recherches in applied mathematics from the University of Rennes I, Rennes, France, in 2007. He is an IEEE Fellow.

$\mathrm{He}$ is a Senior Researcher (Directeur de Recherche) with Inria in Rennes, France, and the scientific leader of the PANAMA research group on sparse audio processing. His research interests include mathematical signal processing, machine learning, approximation theory and statistics. His research focuses on sparse approximation and applications to multichannel audio signal processing, with a particular emphasis in blind audio source separation and compressed sensing. Since 2002 he has been the coordinator of several national, bilateral and european research projects. In 2008 he was elected a member of the steering committee for the international conference ICA on independent component analysis and source separation, and in 2012 he joined the IEEE SPTM Technical Committee. He is the founder of the series of international workshops SPARS on Signal Processing with Adaptive/Sparse Representations. In 2011, he was awarded the Blaise Pascal Award in Applied Mathematics and Scientific Engineering from the SMAI by the French National Academy of Sciences. He has been awarded a starting investigator grant from the European Research Council in 2011. 